\title{
Cardiomiopatia Hipertrófica Apical Associada a Doença Arterial Coronária
}

\author{
Francisco Manes Albanesi F , Justiniano Simões Lopes, José David Aron Diamant, \\ Marcia Bueno Castier, João Alexandre, Rezende Assad, Rosangela Cruz Lyra \\ Rio de Janeiro, RJ
}

\begin{abstract}
Homem de 59 anos, portador de cardiomiopatia hipertrófica apical há 12 anos, apresentou agudização de quadro anginoso. Foi detectada doença arterial coronária com lesão trivascular, e indicada cirurgia de revascularização, com anastomose de artéria mamária e a colocação de duas pontes de veia safena, tendo evoluído com melhora do quadro anginoso e redução da dimensão do átrio esquerdo.
\end{abstract}

\section{Apical Hypertrophic Cardiomyopathy Associated with Coronary Artery Disease}

A fifty-nine year old man, known to have hypertrophic cardiomyopathy, presented worsening of angina. Multivessel coronary artery disease was diagnosed, and he underwent myocardial revascularization (mammary and two safenous grafts were implanted) with good evolution and reduction of left atrium dimension.

Arq Bras Cardiol, volume 71 (n 2$), 139-142,1998$

A cardiomiopatia hipertrófica apical (CMHA) foi inicialmente vista no Oriente ${ }^{1,2}$, porém, passados 21 anos da sua descrição original, é encontrada em todos os continentes, demonstrando sua distribuição universal ${ }^{3}$.

É caracterizada por apresentar no eletrocardiograma (ECG) amplas ondas T, com mais de 10mm de amplitude no plano horizontal e, na ventriculografia esquerda, mostrar a imagem de naipe de espadas devido à acentuada hipertrofia da ponta ${ }^{1,2}$.

No nosso país, são poucas as descrições referentes a esta forma de cardiomiopatia ${ }^{46}$, como também, sua relação com a doença arterial coronária obstrutiva (DAC) ${ }^{7}$.

O objetivo deste relato é relatar a associação da CMHA com a DAC obstrutiva, observada em paciente com diagnóstico de CMHA estabelecido há 12 anos e que desenvolveu doença coronária aterosclerótica.

\section{Relato do Caso}

Homem de 59 anos, branco, com sintomas iniciado em 1985, apresentando dispnéia aos médios esforços e dor precordial. Procurou assistência médica e como o ECG revelava isquemia anterior extensa, a pressão arterial (PA) estava

Universidade do Estado do Rio de Janeiro - UERJ

Correspondência: Francisco Manes Albanesi $F^{o}$ - Serviço de Cardiologia - Av. 28 de Setembro, 77 - $2^{\circ}$ - 20551-030 - Rio de Janeiro, RJ

Recebido para publicação em 6/11/97

Aceito em 3/6/98 elevada (188/104mmHg) e era tabagista de dois maços/dia, foi inicialmente tratado com nitrato e propranolol. Como não se obtivesse melhora, foi submetido a estudo cinecoronariográfico que não revelou $\mathrm{DAC}$, porém demonstrou importante hipertrofia miocárdica restrita à ponta do ventrículo esquerdo (VE). Com o ajuste da dose do betabloqueador para $240 \mathrm{mg} / \mathrm{dia}$, apresentou melhora dos sintomas, permanecendo em acompanhamento ambulatorial.Em abril de 1997, teve seu quadro agravado com o aparecimento de angina do peito aos médios esforços, que cedia com o uso de nitrato sublingual, tendo o quadro se tornado instável, com dor mesmo em repouso e reaparecimento da dispnéia. Foi reavaliado, agora em nossa instituição, tendo os exames revelado: PA de 212/116mmHg, freqüência cardíaca de $68 \mathrm{bpm}$, íctus de $\mathrm{VE}$ no $5^{\circ}$ EIC esquerdo e presença de $4^{\mathrm{a}}$ bulha cardíaca, sem sopros. O ECG mostrou espaço PR de 0,12 s com espessamento inicial no complexo QRS, hipertrofia ventricular esquerda e isquemia anterior extensa, destacando o encontro de amplas ondas $\mathrm{T}$, maiores do que $10 \mathrm{~mm}$ entre $\mathrm{V}_{3}-\mathrm{V}_{4}$ (fig. 1). O ecocardiograma revelou: aorta (Ao) de $3,5 \mathrm{~cm}$; átrio esquerdo (AE) de $4,1 \mathrm{~cm}$; diâmetro diastólico do ventrículo esquerdo(DDVE) de $4,1 \mathrm{~cm}$; diâmetro sistólico do ventrículo esquerdo (DSVE) de 1,9cm; ventrículo direito (VD) de $1,5 \mathrm{~cm}$; septo interventricular (SIV) e parede posterior do VE (ppVE) de 1,2cm; ausência de gradiente intraventricular ao Doppler; com hipertrofia apical e redução do relaxamento do VE (fig. 2). O estudo hemodinâmico demonstrou as seguintes pressões: VE de 160/0/18mmHg e Ao de 160/90mmHg. A ventriculografia 


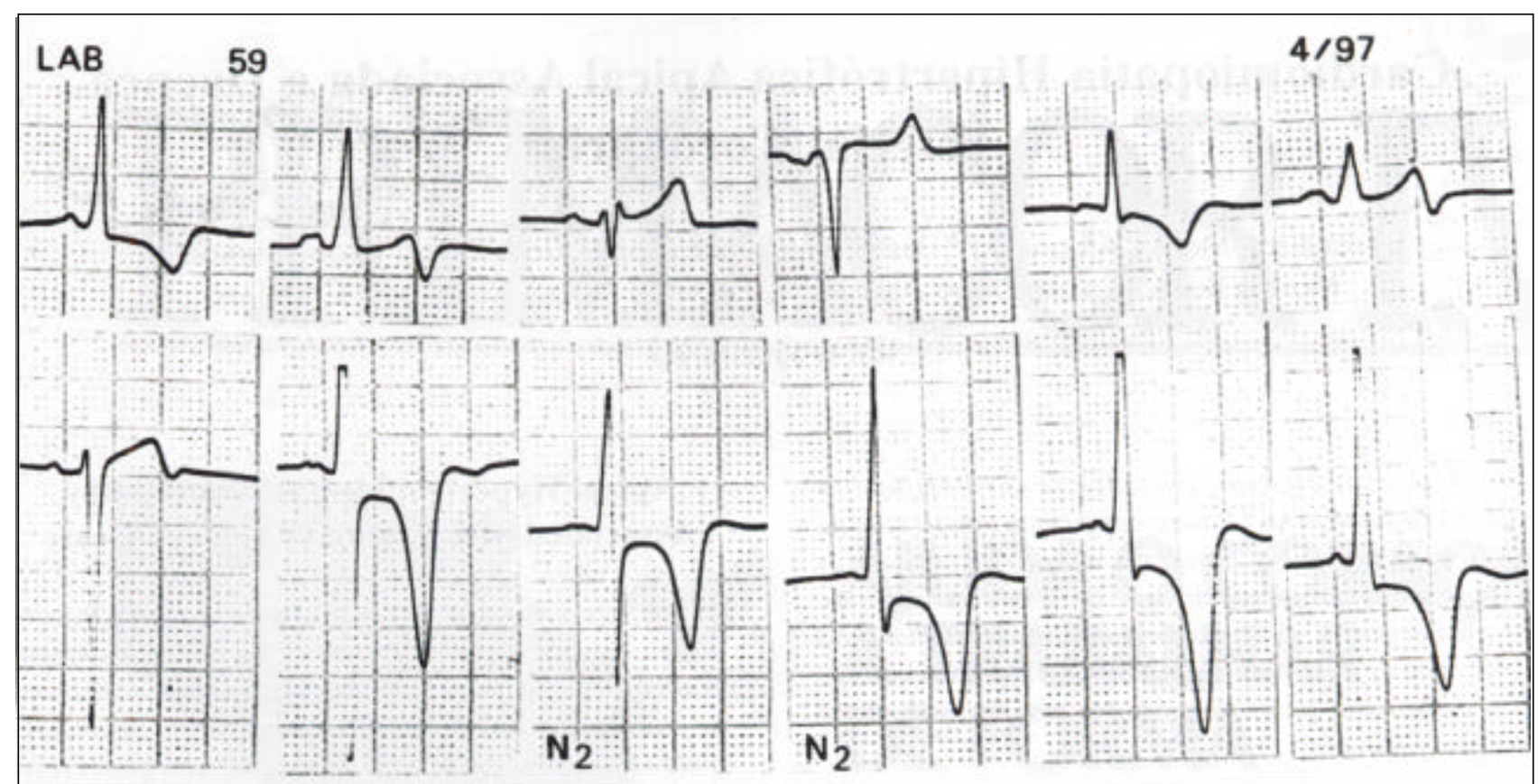

Fig. 1 - O eletrocardiograma revela ritmo sinusal, espaço PR de $0,12 \mathrm{~s}$, hipertrofia ventricular esquerda $\left(\mathrm{S} \mathrm{de}_{\mathrm{V}_{1}}=26 \mathrm{~mm}\right.$ e $\mathrm{R}$ de $\left.\mathrm{V}_{6}=22 \mathrm{~mm}\right)$, isquemia subepicárdica anterior extensa, comonda Temtenda, e amplitude de $18 \mathrm{mmem} \mathrm{V}_{2} \mathrm{eV}_{5} ; 20 \mathrm{em} \mathrm{V}_{3}$ e $24 \mathrm{mmem} \mathrm{V}_{4}$

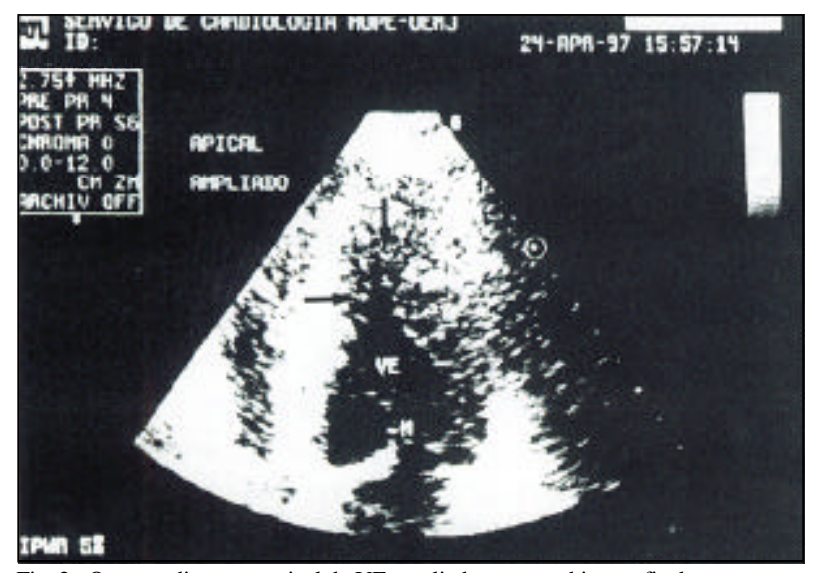

Fig. 2 - O ecocardiograma apical do VE ampliado mostra a hipertrofia da ponta, com aspecto de naipe de espadas.

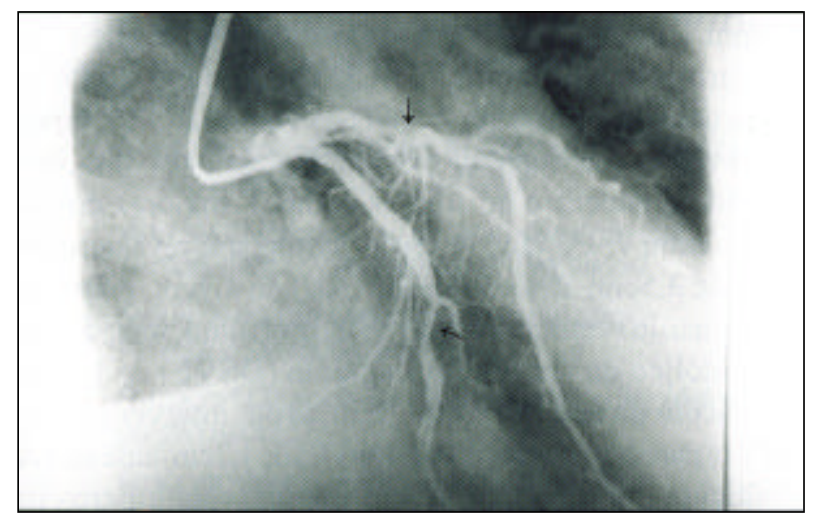

Fig. 3 - A cineventriculografia do ramo esquerdo em obliqua anterior direita, revela lesão no $1 / 3$ proximal $(80 \%)$ da artéria descendente anterior antes da septal principal, com irregularidade parietal no terço médio e distal. A artéria circunflexa também apresenta irregularidade parietal e o ramo marginal pôstero-lateral exibe lesão de $70 \%$ no seu início.

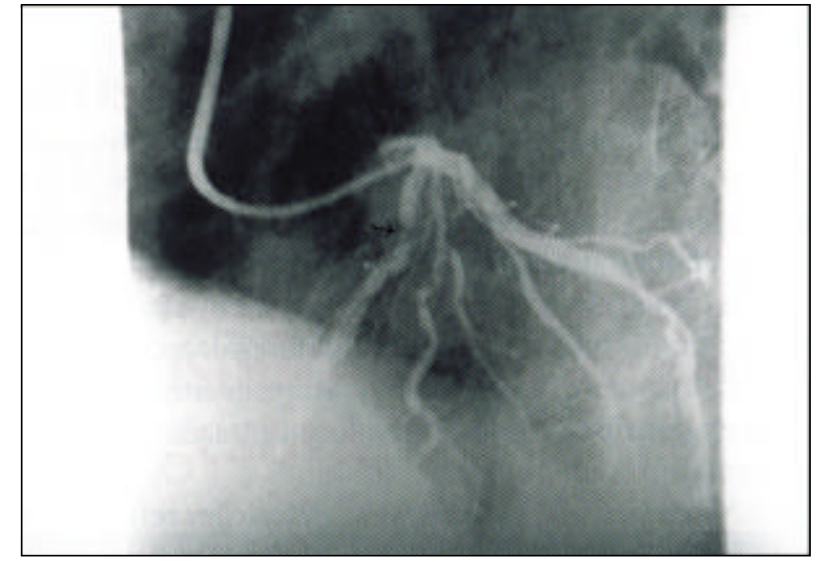

Fig. 4 - A cineventriculografia em obliqua anterior esquerda revelando lesão no 1/3 proximal da artéria descendente anterior, além de irregularidade parietal no restante do seu trajeto.

esquerda revelou grande hipertrofia parietal da ponta com VE de aspecto em naipe de espadas e mobilidade mitral preservada. A cinecoronariografia evidenciou: tronco esquerdo curto e livre de lesão, artéria descendente anterior (ADA) com lesão proximal de $80 \%$ antes da septal principal e com irregularidade parietal, lesão de $75 \%$ no inicio da $1^{\text {a }}$ diagonal; artéria circunflexa com lesão de $70 \%$ na emergência do ramo marginal pôstero-lateral (fig. 3 e 4); artéria coronária direita com oclusão subtotal no $1 / 3$ proximal, lesão de $80 \%$ no $1 / 3$ médio e $70 \%$ após o $2^{\circ}$ ramo marginal (fig. 5). Foi então indicada a cirurgia de revascularização miocárdica, realizada em maio de 1997 com implante de artéria mamária esquerda para a $\mathrm{ADA}$, e pontes de veia safena para a artéria coronária direita e para o $1^{\circ}$ ramo da diagonal, em ato sem circulação extracorpórea. $\mathrm{O}$ paciente teve bom 


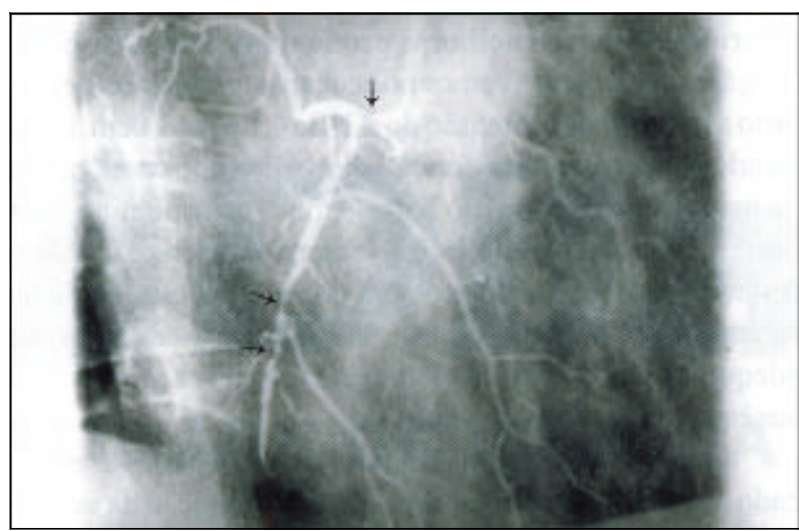

Fig. 5 - A cineventriculografia da coronária direita, em obliqua anterior direita mostra lesão sub-total no $1 / 3$ proximal, lesão localizada de $80 \%$ no $1 / 3$ médio e lesão de $70 \%$ após o $2^{\circ}$ ramo marginal.

pós-operatório e na ocasião da alta o ecocardiograma revelava redução significativa da dimensão do $\mathrm{AE}$ para $3,0 \mathrm{~cm}$. Foi mantido com propranolol, estando atualmente assintomático e com a PA controlada.

\section{Discussão}

A forma apical representa em nossa população 8,34\% dos pacientes com $\mathrm{CMH}^{6}$, sendo mais prevalente o acometimento do VE, porém já observamos três casos com comprometimento do VD sendo em dois isolados e um associado ao $\mathrm{VE}^{5}$.

Poucos são os relatos de casos da forma apical, que evoluíram com infarto agudo do miocárdio (IAM), sem ${ }^{8,9} \mathrm{ou}$ com aterosclerose associada ${ }^{10-12}$. O encontro de angina do peito é comum nos pacientes mais idosos com este tipo de agressão $^{11}$.

A DAC esteve presente em 3,79\% dos nossos pacientes com $\mathrm{CMH}^{7}$, sendo inferior ao referido por vários autores, que estimam sua freqüência entre 7,6 e 19\%, elevada para, aproximadamente, $25 \%$ na faixa etária acima dos 45 anos ${ }^{13-17}$.

Entre 48 casos de CMH necropsiados por Maron e col ${ }^{18}$, $15 \%$ apresentavam áreas de infarto do miocárdio sem comprometimento aterosclerótico, com espessamento da camada média e redução da luz dos pequenos vasos coronários intramiocárdicos, acreditando que, como esta alteração está presente em grande número de pacientes com $\mathrm{CMH}$, ela possa decorrer de componente congênito no processo fundamental da cardiomiopatia.

Na série inicial da CMHA descrita por Sakamoto e cole revista 10 anos após, apenas um único caso entre os 31 seguidos, apresentou instabilização clínica, com aparecimento de angina do peito associada ao consumo excessivo de álcool ${ }^{19}$.

A revisão da literatura referente a associação entre a CMHA e a doença coronária têm poucos casos de exemplificação. Yano e col ${ }^{12}$ referem um paciente de 64 anos com diagnóstico prévio de CMHA, teve infarto do miocárdio de parede inferior, a coronariografia revelou lesão obstrutiva da artéria coronária direita de $75 \%$ no $1 / 3$ proximal e de $90 \%$ no 1/3 médio, estando presente como fator de risco cardiovascular o tabagismo e o nível baixo do HLDcolesterol. Os casos referidos por Çubukçu e col ${ }^{9}$ na Inglaterra, eram de dois pacientes que apresentavam angina em repouso, e que evoluíram com IAM de localização subendocárdica, um com coronariografia mostrando lesão de pequena magnitude no sistema esquerdo e lesão de $50 \%$ na artéria coronária direita, e o outro com coronariograma normal. O paciente referido por Vacek e col ${ }^{10}$ era homem branco de 59 anos, com angina do peito e que, submetido a prova de tolerância ao esforço, apresentou dor, associada a alteração isquêmica na parede inferior, e a cinecoronariografia mostrou lesão de $70 \%$ devido a constrição sistólica por ponte miocárdica na ADA, e lesões obstrutivas de $40 \%$ na artéria coronária direita e de $50 \%$ no $1 / 3$ proximal da artéria descendente posterior. Entre os 23 pacientes vistos em Israel por Keren e col ${ }^{11}$, sete tinham antecedentes de infarto do miocárdio, e 18 referiam angina do peito, seis foram submetidos a cinecoronariografia sendo que em três com antecedentes de infarto, apenas um apresentava pequenas irregularidade da luz dos vasos, porém não sendo significativas para explicar o quadro anginoso. Esta série era composta por pacientes com maior faixa etária $(62 \pm 11 \text { anos })^{11}$. Na série de Webb e col ${ }^{8}$ em Toronto, dos 26 pacientes com CMHA, apenas um apresentou aos 33 anos infarto do miocárdio apical e formação de aneurisma, estava em seguimento ambulatorial desde os 17 anos, tendo ocorrido o desaparecimento das alterações da onda T e surgido elevação persistente de supra desnível do segmento ST-T entre $\mathrm{V}_{3}$ $\mathrm{V}_{6}$. O estudo coronariográfico não evidenciou nenhuma alteração obstrutiva das artérias coronárias, posteriormente desenvolveu arritmia ventricular letal. Observaram, ainda em dois casos, estenoses coronárias $>50 \%$ sem no entanto os pacientes terem desenvolvido infarto do miocárdio.

Outra forma de agressão é da ponte miocárdica, encontrada por Partanen e col ${ }^{20}$ em paciente de 47 anos, com extensa contração segmentar na ADA após o $1^{\circ}$ ramo diagonal, tendo evoluído com formação de aneurisma apical. Esta formação aneurismática geralmente surge após o infarto do miocárdio ${ }^{8}$, pode ser devida a obstrução intracavitária médio-ventricular com gradiente de pressão intraventricular que facilita a formação do aneurisma, pelo aumento da pressão apical e/ ou com a redução da pressão de perfusão coronária desta região ${ }^{21,22}$, porém pode não estar relacionada a áreas de infarto ${ }^{23}$ ou não estar relacionada a lesão obstrutiva coronária ${ }^{8}$.

Foi observado também a associação entre a angina do peito e a presença de fístulas arteriovenosas coronárias em pacientes com CMHA ${ }^{24-26}$, ambas as condições poderão ser responsáveis pelos sintomas anginosos, podendo também as fístulas serem causadoras de roubo coronário. Nos casos com grande defeito na perfusão e resposta positiva ao esforço com tálio, estará indicada a correção cirúrgica das fístulas ${ }^{24-26}$.

Nosso caso é de paciente que sabidamente era portador da forma apical há 12 anos, tendo sido inicialmente diagnosticado como portador de DAC, porém, como não respondeu à terapia com nitrato e propranolol, foi submetido a 
estudo invasivo que mostrou coronárias normais e a hipertrofia da ponta do VE. Posteriormente, foi acompanhado no ambulatório, usando medicação com ação na disfunção diastólica (melhorando o relaxamento do VE) e também com atividade antisquêmica. Era hipertenso e vinha com seus níveis pressóricos controlados, porém ainda persistindo o hábito de fumar; era estressado e não fazia exercícios físicos regulares. Apresentou nos últimos meses o ressurgimento do quadro anginoso, com agravamento de suas crises que passaram a se manifestar até no repouso. Aliado a este quadro, apresentou antes da última internação, quando foi caracterizado a complicação aterosclerótica, descontrole da PA atingindo cifras de até 220/120mmHg. O ECG continuou mostrando isquemia subepicárdica anterior extensa, com amplas ondas T, que permaneceram com a mesma amplitude, com o surgimento do quadro isquêmico obstrutivo. O tratamento cirúrgico foi realizado com a técnica sem emprego de circulação extracorpórea, tendo sido abordado três das principais lesões apresentadas. Como exteriorização das melhoras produzidas pela revascularização miocárdica, tivemos no ecocardiograma pós-operatório a redução da dimensão do $\mathrm{AE}$ de 4,1 para $3,0 \mathrm{~cm}$, mostrando a melhora da disfunção diastólica após o aumento da irrigação ao miocárdio hipertrofiado e isquêmico. Nos pacientes com CMH devemos sempre dar atenção à manifestação da angina do peito que, quando em crescendo ou se manifestando em repouso, deve sempre ser explorada adequadamente, a fim de melhor caracterizar a presença ou ausência da DAC associada.

$\mathrm{O}$ encontro de angina do peito na $\mathrm{CMH}$ pode ser explicado por um dos seguintes mecanismos: redução da velocidade de fluxo e da reserva coronária ${ }^{27}$; existência de alterações nas pequenas artérias intramiocárdicas ${ }^{28}$; no tamanho inadequado das artérias coronárias em relação a massa hipertrofiada ${ }^{29}$; a compressão dos ramos perfurantes septais da ADA devido a hipertrofia septal ${ }^{30}$; pelo espasmo coronário ou decorrer de processo aterosclerótico obstrutivo coronário $^{31}$.

\section{Referências}

1. Sakamoto T, Tei C, Murayama Met al - Giant T wave inversion as manifestation of asymmetrical hypertrophy of the left ventricle. Jpn Heart J 1976; 17: 611-29.

2. Yamaguchi H, Ishimura T, Nishiyama S et al - Hypertrophic nonobstructive cardiomyopathy with giant negative $\mathrm{T}$ waves (apical hypertrophy): ventriculographic and echocardiographic features in 30 patients. Am J Cardiol 1979; 44: 401-12.

3. Albanesi $\mathrm{F}^{\circ} \mathrm{FM}$ - Cardiomiopatia hipertrófica apical. Arq Bras Cardiol 1996; 66: 91-5.

4. Romão N, Anselmo CE, Araujo WB et al - Miocardiopatia hipertrófica apical. Ars Curandi Cardiol 1981; 3: 20-9.

5. Albanesi F. ${ }^{\circ}$ FM, Castier MB, Diamant JDA, Lopes JS, Lopes AS, Ginefra P Cardiomiopatia hipertrófica apical acometendo o VD. Arq Bras Cardiol 1997; 68: 119-24.

6. Albanesi F. ${ }^{\circ} \mathrm{FM}$, Castier MB, Lopes AS, Ginefra P - É a cardiomiopatia hipertrófica apical vista na cidade do Rio de Janeiro similar a encontrada no oriente? Arq Bras Cardiol 1997; 69: 117-23.

7. Albanesi F. ${ }^{\circ} \mathrm{FM}$, Castier MB, Silva TT, Ginefra P - O papel do acometimento isquêmico obstrutivo na evolução da cardiomiopatia hipertrófica do tipo septal assimétrico. Arq Bras Cardiol 1997; 69(suppl. 1): 95.

8. Webb JG, Sasson Z, Rakowski H, Liu P, Wigle ED - Apical hypertrophic cardiomyopathy: Clinical follow-up and diagnostic correlates. J Am Coll Cardiol 1990; 15: 83-90.

9. Çubukçu AA, Scott PJ, Williams GJ - Apical hypertrophic cardiomyopathy presenting as acute subendocardial myocardial infarction. Inter J Cardiol 1993; 38: 329-32.

10. Vacek JL, Davis WR, Bellinger RL, Mckiernan TL - Apical hypertrophic cardiomyopathy in American patients. Am Heart J 1984; 108: 1501-6.

11. Keren G, Belhassen B, Sherez J et al - Apical hypertrophic cardiomyopathy: evaluation by noninvasive and invasive techniques in 23 patients. Circulation 1985; 71: 45-56.

12. Yano A, Saski J, Arakawa K - Combined apical hypertrophic cardiomyopathy and acute myocardial infarctation. Inter J Cardiol 1993; 38: 329-32.

13. Walston A II, Behar VS - Spectrum of coronary artery disease in idiopathic subaortic stenosis. Am J Cardiol 1976; 38: 12-6.

14. Lardani H, Serrano JA, Villami RJ - Hemodynamics and coronary angiography in idiopathic hypertrophic subaortic stenosis. Am J Cardiol 1978; 41: 476-81.

15. Cokkinos DV, Krajcer Z, Leachman RD - Coronary artery disease in hypertrophic cardiomyopathy. Am J Cardiol 1985; 55: 1437-8.

16. Lazzeroni E, Rolli A, Aurier E, Botti G - Clinical significance of coronary artery disease in hypertrophic cardiomyopathy. Am J Cardiol 1992; 70: 499-501.
17. Harjai HJ, Cheirif J, Murgo JP - Ischemia and atherosclerotic coronary artery disease in patients with hypertrophic cardiomyopathy: a review of incidence, pathophysiological mechanisms, clinical implications and management strategies. Coronary Artery Dis 1996; 7: 183-7.

18. Maron BJ; Wolfson JK, Epstein SE, Roberts WC - Intramural (small vessel) coronary artery disease in hypertrophic cardiomyopathy. J Am Coll Cardiol 1986; 8: 545-57.

19. Sakamoto T, Amaro K, Hada $\mathrm{Y}$ et al - Asymmetric apical hypertrophy: ten years experience. Postgrad Med J 1986; 62: 567-70.

20. Partanen J, Kupari M, Heikkilä J, Keto P - Left ventricular aneurysm associated with apical hypertrophic cardiomyopathy. Clin Cardiol 1991; 14: 936-9.

21. Gordon EP, Henderson MA, Rakowski H, Wigle ED - Midventricular obstruction with apical infarction and aneurysm formation. Circulation 1984; 70(suppl II): 145.

22. Wilson P, Marks A, Rastegar H, Manolis AS, Mark Estes III NA - Apical hypertrophic cardiomyopathy presenting with sustained monomorphic ventricular aneurysm. Clin Cardiol 1990; 13: 885-7.

23. Nishiyama S, Ishiwata S, Komiyama $\mathrm{N}$ et al - Long-term prognosis of apical hypertrophic cardiomyopathy. Eur Heart J 1989; 10(suppl I): 413.

24. Lado MP, Pasalodos J, Álvarez LP, Ferro L, Beiras AC - Miocardiopatia hipertrófica apical y fístula arteriovenosa coronaria. Rev Esp Cardiol 1991; 44: 131-3.

25. Chia BL, Yan PC, Choo MH - Combined apical hypertrophic cardiomyopathy and coronary artery-left ventricular fistula. Chest 1988; 94: 219.

26. Lisanti P, Serino W, Petrone M - Fistole multiple coronaria-ventricolo sinistro in paziente com cardiomiopatia ipertrofica apicale: causa insolita doi angina pectoris. G Ital Cardiol 1988; 18: 858-61.

27. Memmola C, Iliceto S, Napoli VF, Cavallari D, Santoro G, Rizzon P - Coronary flow dynamics and reserve assessed by transesophageal echocardiography in obstructive hypertrophic cardiomyopathy. Am J Cardiol 1994; 74: 1147-51.

28. Tanaka M, Fukiwara H, Onodera T et al - Quantitative analysis of narrowings of intramyocardial small arteries in normal hearts, hypertensive hearts and hearts with hypertrophic cardiomyopathy. Circulation 1987; 75: 1130-9.

29. Kimball BP, LiPreti V, Bui S, Wigle ED - Comparison of proximal left anterior descending and circumflex coronary artery dimensions in aortic valve stenosis and hypertrophic cardiomyopathy. Am J Cardiol 1990; 65: 767-71.

30. Pichard AD, Meller J, Teichholz LE, Lipnik S, Gorlin R, Herman MV - Septal perforator compression (narrowing) in idiopathic hypertrophic subaortic stenosis. Am J Cardiol 1977; 40: 310-14

31. Nishimura K, Nosaka H, Saito T, Nobuyoshi M - Another possible mechanism of angina in hypertrophic cardiomyopathy. Circulation 1983; 68(suppl III): 162. 